Planetary Systems in the Universe - Observation, Formation and Evolution

Proceedings IAU Symposium No. 202, (C)2004 IAU

Alan Penny, Pawel Artymowicz, Anne-Marie Lagrange, $\&$ Sara Russell, eds.

\title{
Stability Mechanism of Planetary System of $v$ Andromedae
}

\author{
Hiroshi Kinoshita and Hiroshi Nakai \\ National Astronomical Observatory, Osawa, Mitaka, Tokyo, Japan
}

\begin{abstract}
Three planets are detected around $v$ Andromedae. The stability of Upsilon Andromedae Planetary system is maintained by the co rotation of the pericenters of the two outer planets. If the pericenters of the two outer planets move independently, the planetary system becomes unstable. The corotation of the pericenters is explained by the secular perturbation theory. This corotation is not a secular resonance.
\end{abstract}

Butler et al. (1999) have detected at least three Jovian-mass planets around $v$ Andromedae and determined their masses and orbital elements except the inclination. The stability of this planetary system strongly depends on the orbital elements and the planetary masses. The dynamical behavior of this planetary system is investigated by Laughlin and Adams (1999) and Rivera and Lissauer (2000) with use of a numerical simulation and the planetary system is found to be stable by the alignment of the pericenters of the outer two planets, which prevents close approaches between them.

We integrated the planetary system of $v$ Andromedae with use of the orbital elements determined by Butler et al.(1999). The left panel of Figure 1 shows the semi-major axes $(a)$, eccentricities $(e)$, and the difference of the pericenter longitudes $(\theta)$ of the outer two planets and the pericenters of two planets moves together and the close approach does not occur in spite of their large eccentricities and the system is quite stable. Then we integrated a fictitious planetary system, in which the initial pericenter longitude of the planet $c$ is displaced by 180 degrees from the observed position and show the result in the right panel of Figure 1. In this case both the pericenters move independently and the close approaches take place frequently and finally the planet $c$ is ejected from the system.

Now we interpret the corotation or the alignment of the pericenters with use of the secular perturbation theory. We assume the outer two planets are moving the same plane and neglect the innermost planet $b$, which is small and well separated from the outer planets. The number of degree of freedom of this system is four with the Hamiltonian

$$
F=F\left(a_{c}, a_{d}, e_{c}, e_{d}, \varpi_{c}, \varpi_{d}, \lambda_{c}, \lambda_{d}\right),
$$

where $\lambda_{c}$ and $\lambda_{d}$ are the mean longitudes, $\varpi_{c}$ and $\varpi_{d}$ are the pericenter longitudes, and the subscripts $c, d$ represents the planet $c$ and $d$. After the elimination of short periodic terms the degree of freedom is reduced to one, since $\varpi_{c}$ and $\varpi_{d}$ do appear only in the form of $\varpi_{c}-\varpi_{d}$. The Hamiltonian after the elimination of short periodic terms takes the form $F^{*}=F^{*}\left(e_{c}, e_{d}, \theta\right), \theta=\varpi_{c}-\varpi_{d}$. Since 

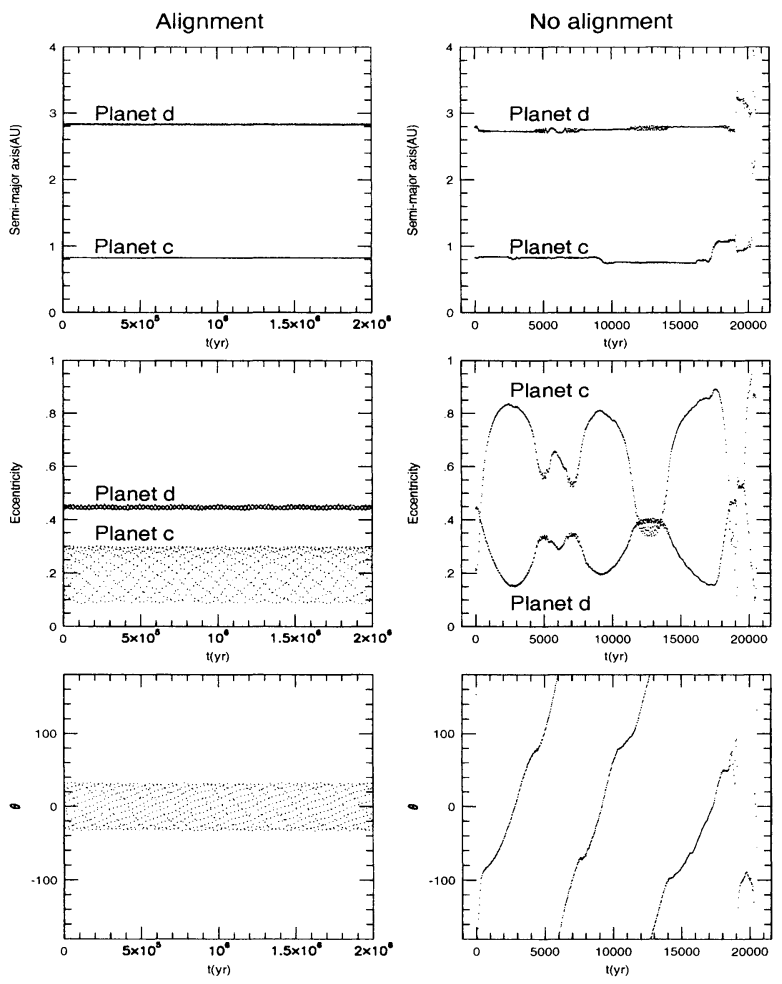

Figure 1. Orbital elements: semi-major axes, eccentricities, and the difference of the pericenter longitudes of the planets $c$ and $d$. In the alignment case the pericenters corotate and in the no alignment case they moves independently and the system soon becomes unstable.

the eccentricities are related through the angular momentum integral,

$$
m_{c} \sqrt{a_{c}\left(1-e_{c}^{2}\right)}+m_{d} \sqrt{a_{d}\left(1-e_{d}^{2}\right)}=\text { const. }
$$

we can draw the contour map of $F^{*}$ in the phase plane of $\left(\theta, e_{c}\right)$, see Figure 2. From this contour we know the global motion of the system without solving the equations of motion. The dots in Figure 2 are the result of the numerical integration (the left panel of Figure 1 ) and are in good agreement with the secular perturbation theory.

Now we apply the linear secular perturbation theory, in which the eccentricities are small and the Hamiltonian takes the form,

$$
F^{*}=G m_{c} m_{d}\left[N_{12}\left(a_{c}, a_{d}\right)\left(e_{c}^{2}+e_{d}^{2}\right)-2 P_{12}\left(a_{c}, a_{d}\right) e_{c} e_{d} \cos \left(\varpi_{c}-\varpi_{d}\right)\right] .
$$

In this case the equations of motion become a linear differential equation with constant coefficients. At first we solve the secular equation for this linear equation and get two eigen values, $\omega_{1}=30 " .59 / y, \omega_{2}=200 " .35 / y$ and corresponding 


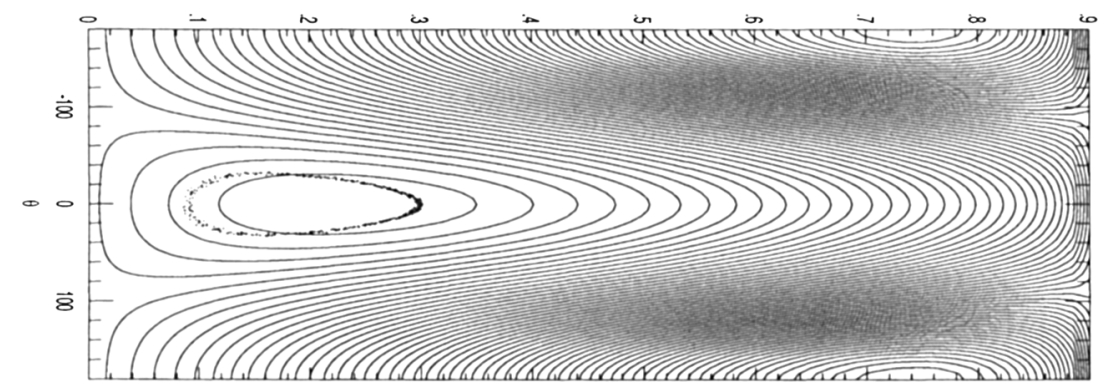

Figure 2. Contour map of the Hamiltonian after the elimination of the short periodic terms: the dots are the solution from the numerical integration for the alignment case of Fig. 1

periods $42000 \mathrm{y}$ and $6500 \mathrm{y}$. Solutions for the initial values: $e_{c 0}=0.22, \varpi_{c 0}=$ $219 .^{\circ} 2, e_{d 0}=0.44, \varpi_{d 0}=245 .^{\circ} 9$ (Butler et al. (1999)) are

$e_{c} \cos \varpi_{c}=0.1928 \cos \alpha_{1}+0.0955 \cos \alpha_{2}, e_{c} \sin \varpi_{c}=0.1928 \sin \alpha_{1}+0.0955 \sin \alpha_{2}$ $e_{d} \cos \varpi_{d}=0.4404 \cos \alpha_{1}-0.0078 \cos \alpha_{2}, e_{d} \sin \varpi_{d}=0.4404 \sin \alpha_{1}-0.0078 \sin \alpha_{2}$, where $\alpha_{1}=\omega_{1} t+244 .^{\circ} 9, \alpha_{2}=\omega_{2} t+158 .{ }^{\circ} 2$.

Since the component of the eigen-frequency $\omega_{1}$ is dominant for both planets, the pericenter longitudes take the following form

$$
\varpi_{c}=\alpha_{1}+(\text { periodic }), \varpi_{d}=\alpha_{1}+(\text { periodic }),
$$

which means that the pericenters corotate and the difference of the pericenter longitudes is periodic. Since the eigen values are not close, this corotation is not a secular resonance. A secular resonance occurs in the case that two eigen frequencies become nearly equal.

\section{References}

Butler, R. P., Marcy, G. W., Fischer, D. A., Brown, T. M., Contos, A. R., Korzennik, S. G., Nisenson, P. \& Noyes, R. W. 1999, ApJ, 526, 916

Laughlin, G., \& Adams, F. C. 1999, ApJ, 526, 881

Rivera, E. J., \& Lissauer, J. J. 2000, ApJ, 530, 454 